\title{
Video Article \\ Automated System for Single Molecule Fluorescence Measurements of Surface-immobilized Biomolecules
}

\author{
Nicolas Di Fiori ${ }^{1}$, Amit Meller $^{1,2}$ \\ ${ }^{1}$ Physics Department, Boston University \\ ${ }^{2}$ Department of Biomedical Engineering, Boston University \\ Correspondence to: Amit Meller at ameller@bu.edu \\ URL: http://www.jove.com/video/1542 \\ DOI: doi:10.3791/1542
}

Keywords: Cellular Biology, Issue 33, single molecule FRET, DNA, surface immobilization, microfluidics, confocal microscope

Date Published: 11/2/2009

Citation: Di Fiori, N., Meller, A. Automated System for Single Molecule Fluorescence Measurements of Surface-immobilized Biomolecules. J. Vis. Exp. (33), e1542, doi:10.3791/1542 (2009).

\section{Abstract}

Fluorescence Resonance Energy Transfer (FRET) microscopy has been widely used to study the structure and dynamics of molecules of biological interest, such as nucleic acids and proteins. Single molecule FRET (sm-FRET) measurements on immobilized molecules permit long observations of the system -effectively until both dyes photobleach- resulting in time-traces that report on biomolecular dynamics with a broad range of timescales from milliseconds to minutes. To facilitate the acquisition of large number of traces for statistical analyses, the process must be automated and the sample environment should be tightly controlled over the entire measurement time ( 12 hours). This is accomplished using an automated scanning confocal microscope that allows the interrogation of thousands of single molecules overnight, and a microfluidic cell that permits the controlled exchange of buffer, with restricted oxygen content and maintains a constant temperature throughout the entire measuring period. Here we show how to assemble the microfluidic device and how to activate its surface for DNA immobilization. Then we explain how to prepare a buffer to maximize the photostability and lifetime of the fluorophores. Finally, we show the steps involved in preparing the setup for the automated acquisition of time-resolved single molecule FRET traces of DNA molecules.

\section{Video Link}

The video component of this article can be found at http://www.jove.com/video/1542/

\section{Protocol}

\section{1 - Assembling the microfluidic cell}

The microfluidic cell is made by fusing a patterned, $2 \mathrm{~mm}$ thick, polydimethylsiloxane (PDMS) disk to a freshly cleaned quartz cover slip. The PDMS disk contains four $30 \mu \mathrm{l}$ rectangular chambers that are accessed by inlet and outlet flexible capillaries connected to a buffer reservoir and a syringe pump, respectively, for the controlled flow of buffer. The flow cell is supported on its back side by a glass window and placed on a custom-made cell holder containing a water cooled thermoelectric element to regulate temperature.

1. To make the patterned PDMS disk, mix 10 parts silicone elastomer base with 1 part curing agent, mix well and leave degassing for 1 hour in vacuum.

2. Gently pour the elastomer mix onto the microfluidic cell mold. In our case, this is a 1 " silicon wafer with four strips $\left(1 \times 10^{4} \times 1 \times 10^{3} \times 300 \mu \mathrm{m}\right)$ made from negative photoresist. Leave this to cure on a hot plate at $70^{\circ} \mathrm{C}$ for two hours.

3. Carefully peel the cured PDMS disk from the mold using a razor blade. Fuse the disk onto a 1 " glass window (containing $8 \times 2 \mathrm{~mm}$-diameter pre-drilled holes at both ends of the channels) by plasma oxidizing both surfaces for 30 seconds. Fuse the glass windows onto the flat side of the PDMS disk (the side not containing the channels).

4. Insert a 2-inch long flexible fused silica capillary tubing through each glass window's hole until it reaches the channel. Inserting a needle first and then following with the capillary may facilitate this process. Seal the glass window holes using a fast curing silicone casting compound such as Kwik-Cast.

5. Rinse the channels with ethanol and water, and plasma-activate the cell together with a freshly cleaned, 1-inch circular quartz cover slip for 30 seconds. We suggest cleaning this cover slip using piranha*. Fuse the cover slip to the side of the cell containing the channels, sealing the chambers.

6. Leave the assembled cell to cure overnight at room temperature.

* Piranha is a solution of $\mathrm{H}_{2} \mathrm{O}_{2}$ and $\mathrm{H}_{2} \mathrm{SO}_{4}$ in 1:2.5 proportions. To clean the cover slips, immerse them in piranha at $90^{\circ} \mathrm{C}$ for 20 min. 


\section{2 - Activating the surface for molecule immobilization}

For nucleic acid studies, the simplest surface immobilization scheme consists of first coating a glass or quartz cover slip with biotinylated bovine serum albumin (BSA) and then with streptavidin. This allows the biotinylated sample to be immobilized with high specificity for days at room temperature.

1. Connect flexible tubing to the capillaries for easy buffer injection using a syringe and needle.

2. Inject a solution of $0.1 \mathrm{mg} / \mathrm{ml}$ of biotinylated BSA in buffer A (10 mM TRIS- $\mathrm{HCl}, \mathrm{pH} 8.0,50 \mathrm{mM} \mathrm{NaCl}$, filtered using $0.02 \mu \mathrm{m}$ filters) into the channel and incubate for at least $30 \mathrm{~min}$.

3. Flow 300-500 $\mu \mathrm{l}$ of buffer A to remove any free biotinylated BSA from the channel, being careful not to trap any air bubbles inside the chamber.

4. Inject a solution of $0.1 \mathrm{mg} / \mathrm{ml}$ of streptavidin in buffer A and incubate for $15 \mathrm{~min}$. Then repeat Step 3.

5. Flow through a 3-5 pM solution of the biotinylated sample. Perform a surface scan to check the coverage of fluorescent molecules. If necessary, increase the sample concentration to obtain better coverage. Incubate for 10 min and repeat Step 3.

The microfluidic cell is mounted on a motorized $x$-y stage allowing coarse (up to $25 \mathrm{~mm}$ ) motion using optically encoded DC motors, and fine (1 $\mathrm{nm}$ ) motion using two closed-loop piezo actuators (Physik Instrumente model M-014 or similar). This can be done either before or after activating the surface for sample immobilization.

\section{3 - Preparing the Imaging Buffer (IB)}

The IB consists of an enzymatic oxygen scavenging system and a triplet quencher, such as Trolox. Since the IB is flushed through constantly overnight, at least $10 \mathrm{ml}$ should be prepared in advance.

1. Prepare $10 \mathrm{ml}$ of $0.8 \% \mathrm{w} / \mathrm{v}$ D-glucose, at least $35 \mathrm{mM}$ TRIS- $\mathrm{HCl} \mathrm{pH} 8.0$ and $50 \mathrm{mM} \mathrm{NaCl}$ in deionized water. The higher concentration of buffer is important for two reasons: dissolving Trolox will acidify the solution, and the enzymatic reaction will also lower the pH of the solution over time. Since this solution has a long shelf life it can be stored as a stock solution.

2. Dissolve $5 \mathrm{mg}$ of Trolox in the buffer prepared in Step 1 by vortexing for a couple of minutes and then shaking for $>10 \mathrm{~min}$. Trolox is not very soluble in water at $\mathrm{pH}>7$, so do not rush this step. Filter the solution using a $0.2 \mu \mathrm{m}$ filter and leave degassing in vacuum for 10 minutes.

3. Prepare the "gloxy" enzymatic solution by mixing $60 \mu \mathrm{l}$ water, $20 \mu \mathrm{l}$ of $5 \mathrm{X}$ buffer $\mathrm{A}, 20 \mu \mathrm{l}$ of catalase, 1 mg glucose oxidase and $100 \mu \mathrm{l}$ of 10 $\mathrm{mg} / \mathrm{ml} \mathrm{BSA}$. Filter the solution using a $0.22 \mu \mathrm{m}$ centrifuge filter.

4. Gently mix the buffer from Step 2 with the "gloxy" solution from Step 3 avoiding the formation of air bubbles.

5. Flow the IB through the channel at a constant rate of $5 \mu \mathrm{l} / \mathrm{min}$ using a mechanical syringe pump to control the flow rate. Bubble argon gas into the IB reservoir to prevent atmospheric oxygen from re-dissolving into the buffer.

The surface scanning, molecule localization and acquisition of single molecule intensity traces is controlled by a LabView program that permits automated data collection ${ }^{1}$. The program scans $20 \times 20 \mu \mathrm{m}^{2}$ areas at $0.2 \mu \mathrm{m}$ resolution, with a rate of $0.1 \mu \mathrm{m} / \mathrm{ms}$. Data is immediately processed to yield intensity-weighted locations of all pixels above the background counts. Once the immobilized molecules are found, they are moved one by one into the confocal volume and the intensities of the donor and acceptor fluorophore are recorded as a function of time until both fluorophores photobleach. The stage is then programmed to move to a new origin $100 \mu \mathrm{m}$ away, and the scanning process is repeated.

\section{4 - Representative Results}

Below are some images showing the assembled microfluidic cell before surface activation and after being mounted on the microscope ready for molecule immobilization. If the channel is activated successfully, surface scans should be similar to the scans depicted below showing emission of the donor dye (green) and acceptor (red) after direct donor excitation. These molecules are moved one by one into the probing volume and the fluorescence is recorded over time until both dyes photobleach, as shown in the single molecule traces. From traces like these one can obtain the FRET efficiency which informs on the instantaneous donor-acceptor separation, which in turn is used to explore the structure and dynamics of molecules of biological interest. 


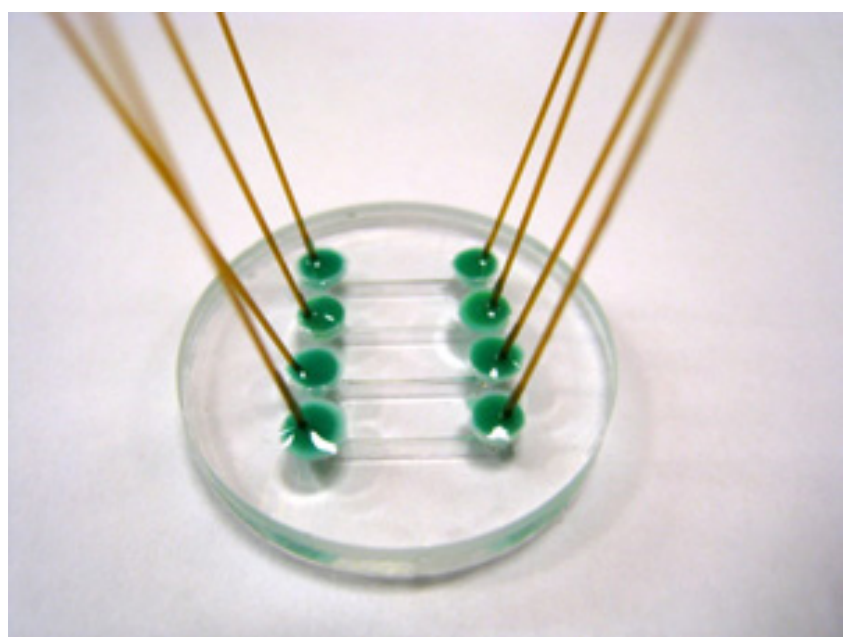

Figure 1: Microfluidic cell with four channels, each with inlet/outlet capillaries. Please click here to see a larger version of figure 1.

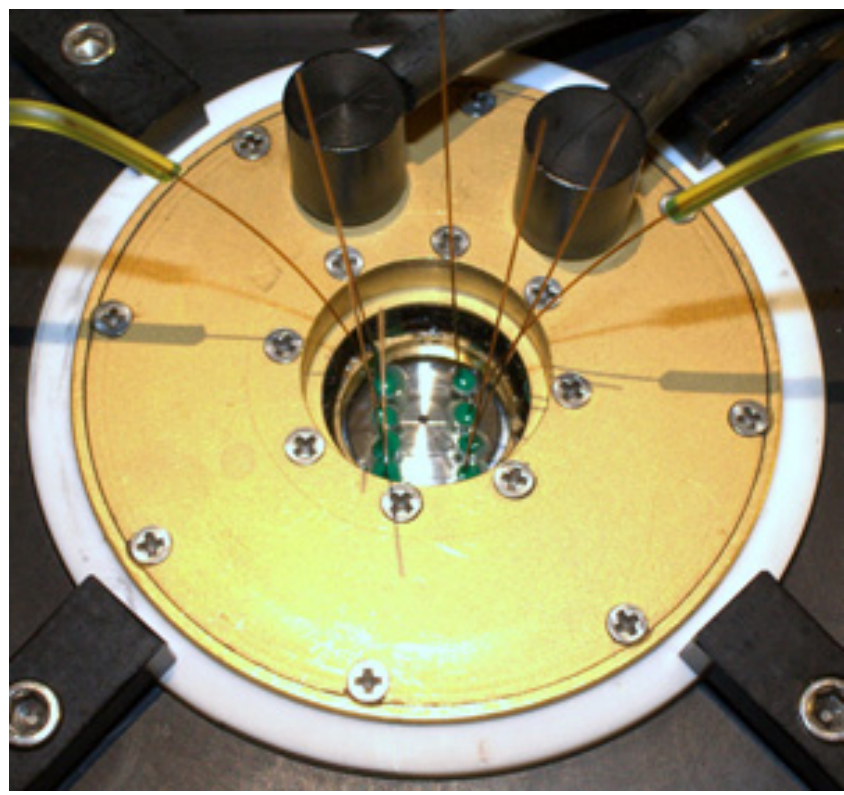

Figure 2: Cell mounted on the microscope ready for measurements. Please click here to see a larger version of figure 2.

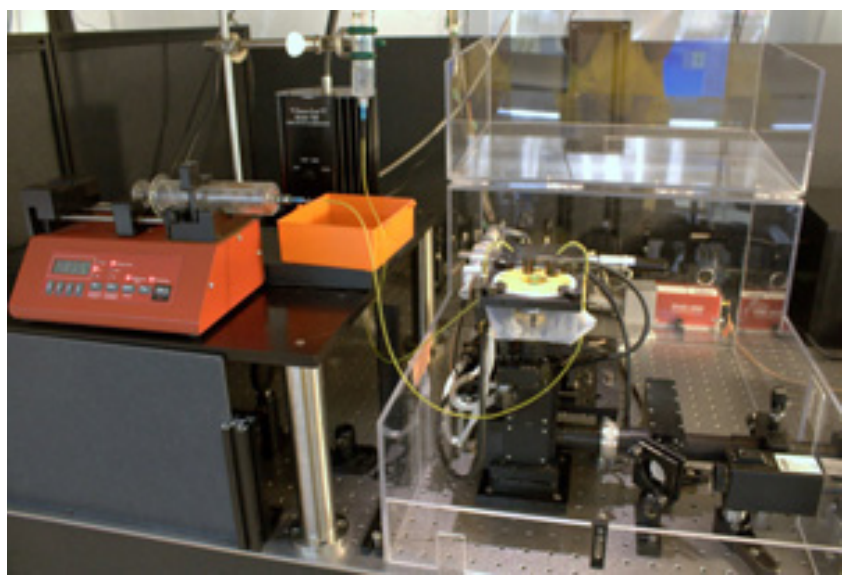

Figure 3: Setup for sm-FRET measurements. Please click here to see a larger version of figure 3. 

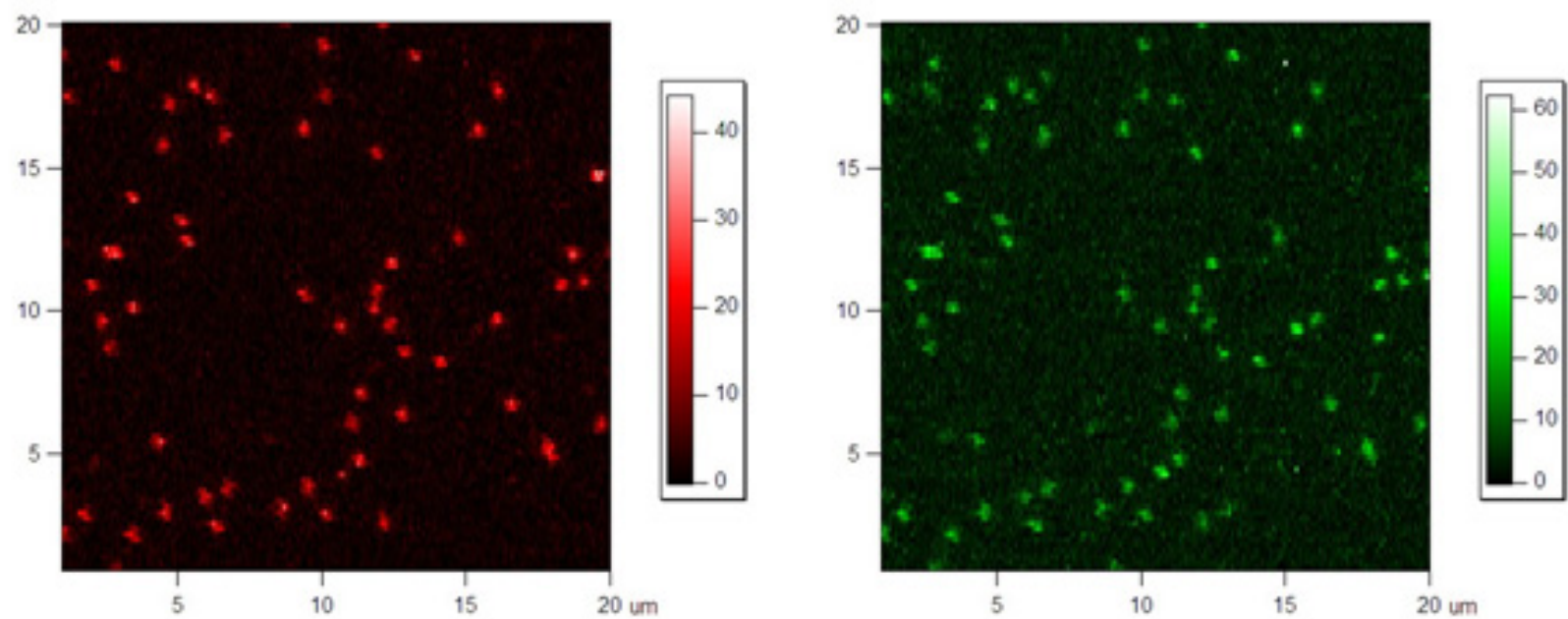

Figures 4 and 5: Red and green channel of a surface scan of immobilized FRET molecules excited with green laser. Please click to see a larger version of figure 4 , or figure 5.
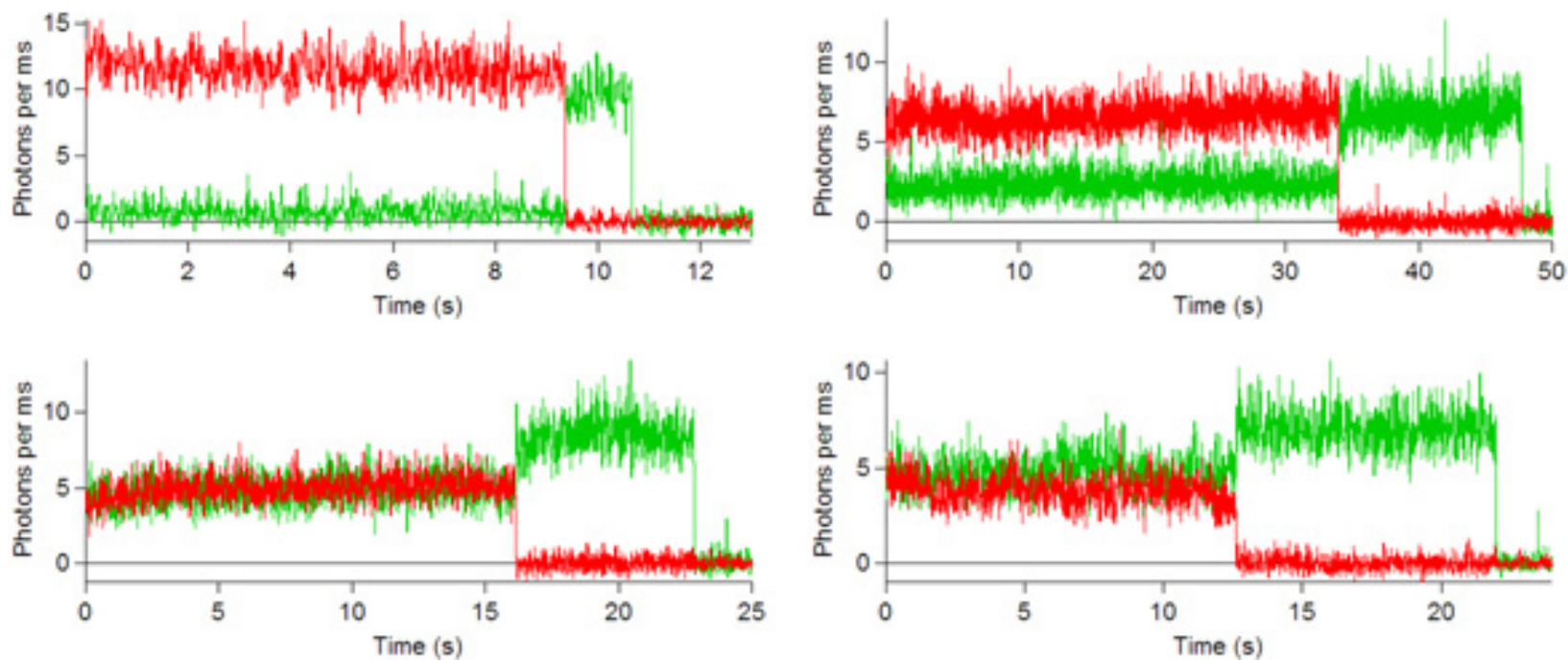

Figures 6, 7, 8, and 9: Intensity trajectories of donor (green) and acceptor (red) fluorophores labeled to a DNA molecule 8, 10, 16 and 18 basepairs apart. Please click to see a larger version of figure 6 , figure 7 , figure 8 , or figure 9.

\section{Discussion}

Although the above protocol has been optimized for our particular system and for a specific choice of dyes (TMR and ATTO647N), it is by no means the only proved method. Recently, an alternative oxygen scavenging system consisting of protocatechuic acid (PCA)/ protocatechuate-3,4-dioxygenase (PCD) was shown to increase the photostability of certain dyes ${ }^{4}$. Likewise, other triplet-state quenchers such as $\beta$-mercaptoethanol (BME) can be used instead of Trolox, although these might not suppress long-lasting blinking of some dyes, in particular Cy $5^{5}$.

Although immobilization via biotin-streptavidin-biotinylated bovine serum albumin (BSA) is the preferred choice for nucleic acids studies, a polyethylene glycol (PEG)-coated surface is better suited for studies of proteins since it prevents non-specific adhesion ${ }^{1}$. Additionally, subdiffraction-limited size vesicles can be used to encapsulate the biomolecule of interest in cases where it can be affected by surface interactions ${ }^{6}$.

In this article we have used a confocal setup equipped with silicone avalanche photodiodes to acquire the fluorescence intensity traces. This protocol works equally well with Total Internal Reflection Fluorescence microscopes (TIRF) equipped with CCD cameras. Regardless of the microscopy used, sm-FRET can inform on spatial resolutions approaching the angstrom when the donor and acceptor signal are properly corrected ${ }^{7}$.

\section{Acknowledgements}

We thank Chandran Sabanayagam for his help in developing the system, Joshua Edel for advice on designing the microfluidic device and the staff at the Rowland Institute at Harvard University for machining parts of the setup. We acknowledge useful discussions with members 
of Meller's group at the Rowland Institute and at Boston University, and members of T. Ha's group at UIUC for helpful information. Finally, we appreciate the financial support from the National Science Foundation (PHY-0701207) and the National Institute of Health (GM075893).

\section{References}

1. Selvin, P.R \& Ha, T. Single-Molecule Techniques, a Laboratory Manual. CSHL Press (2008).

2. Sabanayagam, C.R, Eid, J.S. \& Meller, A. High-throughput scanning confocal microscope for single molecule analysis. Applied Pys. Letters. 84-7, 1216-1218, (2004).

3. Sabanayagam, C.R, Eid, J.S. \& Meller, A. Using fluorescence resonance energy transfer to measure distances along individual DNA molecules: Corrections due to nonideal transfer. J. Chem. Phys. 122, 61103-61105, (2004).

4. Echeverr a Aitken, C., Marshall, R.A., Puglisi, J.D. An Oxygen Scavenging System for Improvement of Dye Stability in Single-Molecule Fluorescence Experiments. Biophysical Journal 94, 1826-1835 (2008).

5. Rasnik, I. ,McKinney, S.A., Ha, T. Nonblinking and long-lasting single molecule fluorescence imaging. Nature Methods 3-11 891-893 (2006).

6. Boukobza, E., Sonnenfeld, A., Haran, G. Immobilization in Surface-Tethered Lipid Vesicles as a New Tool for Single Biomolecule Spectroscopy. J. Phys. Chem. B 105, 12165-12170 (2001).

7. Di Fiori, N., Meller, A. (in preparation) 